Z. Klin. Chem. Klin. Biochem.

12. Jg. 1974 , S. $539-542$

\title{
Aktivitätsmessungen der Aldolase-Isoenzyme in Zwillingen im Vergleich mit einer Stichprobe gesunder Personen
}

\author{
Von H. W. Goedde, Heide-G. Benkmann, L. Hirth, \\ Institut für Humangenetik (Direktor: Prof. Dr. H. W. Goedde) der Universität Hamburg,
}

\author{
H. Lang und $U$. Würzburg, \\ Biochemische Forschung, E. Merck, Darmstadt
}

(Eingegangen am 29. Mai/30. August 1974)

\begin{abstract}
Bei einer Stichprobe von über 100 gesunden, nicht verwandten Personen sowie bei 20 monozygoten und 20 dizygoten Zwillingspaaren wurde die Aktivität der Gesamtaldolase sowie die der Isoenzyme Aldolase A und Aldolase B bestimmt. Die jeweiligen spezifischen Aktivitätswerte sind bei den eineiigen Zwillingspaaren innerhalb der Fehlergrenze identisch. Bei zweieiigen Zwillingen sind signifikante Unterschiede zu verzeichnen. Bezüglich der Zwillingsuntersuchungen wurden analoge Ergebnisse für die Polymorphismen Phosphoglucomutase locus 3, $\alpha_{1}$-Antitrypsin, Alanin-Transaminase, 3.Komponente des Komplements und Transferrin beschrieben (Phänotypenbestimmung).
\end{abstract}

\section{The activity of aldolase isoenzymes in twins in comparison with normal healthy persons}

The activity of total aldolase and of the isoenzymes aldolase $\mathbf{A}$ and aldolase $\mathbf{B}$ was determined in a sample of more than 100 healthy unrelated persons as well as in pairs of 20 monozygotic and 20 dizygotic twins. In monozygotic twin pairs the specific activity is almost identical, whereas significant differences were observed in dizygotic twins. Analogous results with respect to these twin studies were obtained by estimating the phenotypes of the phosphoglucomutase locus $3, \alpha_{1}$-antitrypsin, alanine transaminase, the 3 rd component of complement and transferrin.

Die Aldolase (Fructose-1,6-diphosphat- $D$-glycerinaldehyd-3-phosphat-Lyase, EC 4.1.2.13.) katalysiert die Reaktion Fructose-1.6-diphosphat $\rightleftharpoons$ Dihydroxyacetonphosphat $+D$-Glycerinaldehyd-3-phosphat. Der höchste Aldolasegehalt wurde in der Skelettmuskulatur (Aldolase A) gefunden. In der Leber kommt Aldolase B vor. Die dritte Form, Aldolase C, wurde im Gehirn entdeckt. Im menschlichen Serum von gesunden Personen ist die Aldolase-Enzymaktivität relativ niedrig. Hierüber wurde bisher noch wenig berichtet (1). Unsere Bestimmungen der Aldolase-Aktivitäten bei einer Stichprobe von über 100 nicht verwandten Personen ergaben, daß die Aldolase-Aktivitäten bei Gesunden intraindividuell konstant und interindividuell unterschiedlich sind. Um zu klären, inwieweit die Aktivitätswerte genetisch bedingt sind oder durch äußere Faktoren modifiziert werden können, wurden die im folgenden beschriebenen Zwillingsuntersuchungen durchgeführt.

\section{Material und Methoden}

Untersucht wurden über 100 nicht verwandte Personen sowie 20 eineiige und 20 zweieiige Zwillingspaare im Alter von 12-13 Jahren aus dem Hamburger Raum; das Geschlecht wurde nicht berücksichtigt. Die Zuordnung zur Gruppe der eineiigen und der zweieiigen Zwillingspaare erfolgte mit Hilfe des erbbiologisch-anthropologischen Ähnlichkeitsvergleichs, den Blut- gruppen sowie der Polymorphismen Alanin-Transaminase nach Goedde \& Benkmann (2), Phosphoglucomutase locus 3 ( $\mathrm{PGM}_{3}$ ) nach Goedde \& Stahn (3), $\alpha_{1}$-Antitrypsin $\left(\alpha_{1}\right.$ at) nach Fagerhol \& Laurell (4) und 3. Komponente des Komplements (C3) und Transferrin (Tf) nach Teisberg (5).

Die Gesamtaldolase-Aktivität wurde mit der Boehringer Testcombination Aldolase No. 15974 am Eppendorf-Photometer bei $37^{\circ} \mathrm{C}$ und $366 \mathrm{~nm}$ getestet.

Zur Bestimmung der Isoenżyme Aldolase A und B kam die Vorschrift von Würzburg et al. (6) zur Anwendung. Die Aldolase-Aktivitäten dès Serums wurden vor und nach Präzipitation mit Isoenzym-spezifischen Antikörpern (Antiseren für Aldolase A und B von Merck) gemessen.

\section{Ergebnisse und Diskussion}

Feissli et al. (1) beobachteten bei 48 Personen Aktivitäten der Gesamtaldolase von 0,5-3,1 U/1. Isoenzyme wurden von Feissli nicht bestimmt. In unserem Kollektiv, das sich aus einer Stichprobe von über 100 nicht verwandten Personen aus der Hamburger Bevölkerung zusammensetzt, finden wir etwas höhere Werte von 0,9-4,1 U/1. Bei der Untersuchung der Isoenzyme erhielten wir für Aldolase A 0,6-3,2 U/1, das entspricht etwa 57-86\% der Gesamtaktivität; für Aldolase B beobachteten wir Werte von $0,2-1,0 \mathrm{U} / 1$, also ungefähr 12-47\% der Gesamtaktivität. Aldolase C wurde nicht bestimmt, da sie nach bisherigen Kenntnissen im Blut 
Gesunder nicht in meßbaren Größenordnungen vorkommt. Die beobachteten Aktivitätswerte der Aldolase und Aldolase-Isoenzyme bei Zwillingen liegen im Normalbereich.

Die Aktivitäten der Gesamtaldolase bei Zwillingen zeigt Abbildung 1; die Korrelation der Meßwerte zwischen Zwilling a und Zwilling $b$ ist dargestellt. Die Werte der eineiigen Zwillinge (EZ) sind oberhalb, die der zweieiigen Zwillinge (ZZ) unterhalb der Identitätsgeraden aufgetragen. Die gestrichelten Linien bezeichnen den 2s-
Bereich der Bestimmungsmethode. Die Unterschiede der Aldolase-Aktivitäten zwischen Zwilling a und $\mathrm{b}$ bei EZ liegen innerhalb der Fehlergrenze. Bei ZZ sind signifikante Unterschiede $\mathrm{Zwischen} \mathrm{Zwilling} \mathrm{a} \mathrm{und} \mathrm{Zwilling}$ $b$ vorhanden.

Abbildung 2 zeigt die Aktivitäten von Aldolase $A$ und Aldolase $B$ bei $Z$ willingen. Die Darstellung erfolgte wie bei den Gesamtaldolase-Werten. Es ergibt sich, daß bei der Aktivität von Aldolase A und Aldolase B die Meßwerte bei Paaren vor EZ ebenfalls innerhalb der Fehler-

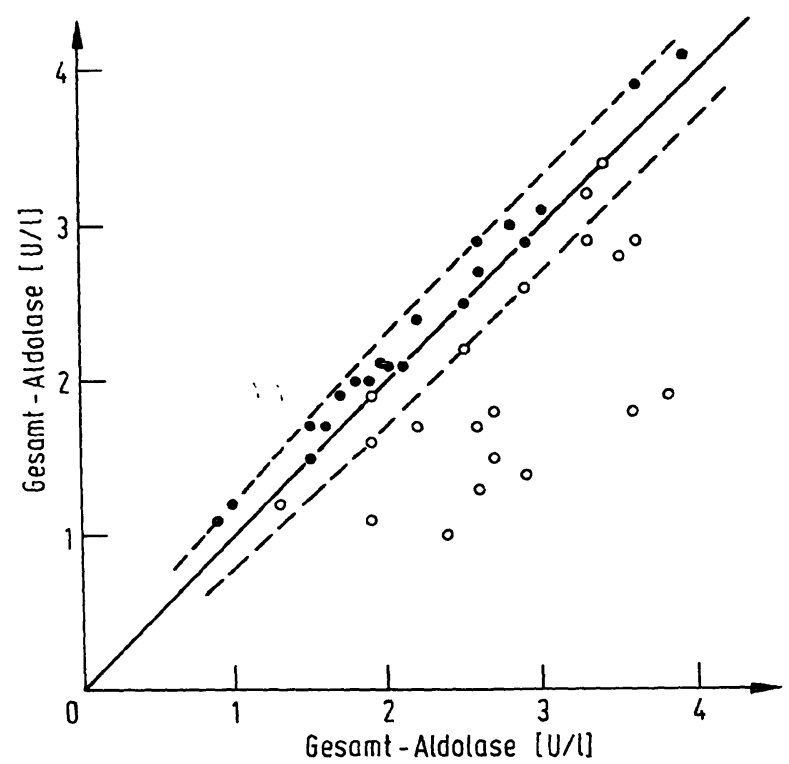

Abb. 1. Aktivität der Gesamt-Aldolase (U/1) bei $Z$ willingspaaren - Eineiige Zwillinge (EZ),

$\circ$ Zweieiige Zwillinge (ZZ)

Korrelation der Aktivitäten bei $\mathbf{Z}$ willing a und Zwilling $b$. - Identitätsgerade (Aktivitäten Zwilling $\mathrm{a}=\mathrm{Z}$ willing $\mathrm{b}$ ). - - 2 s-Bereich der Analysenmethode.

Auftragung der Werte für EZ oberhalb der Identitätsgeraden; für $\mathrm{ZZ}$ unterhalb der Identitätsgeraden.

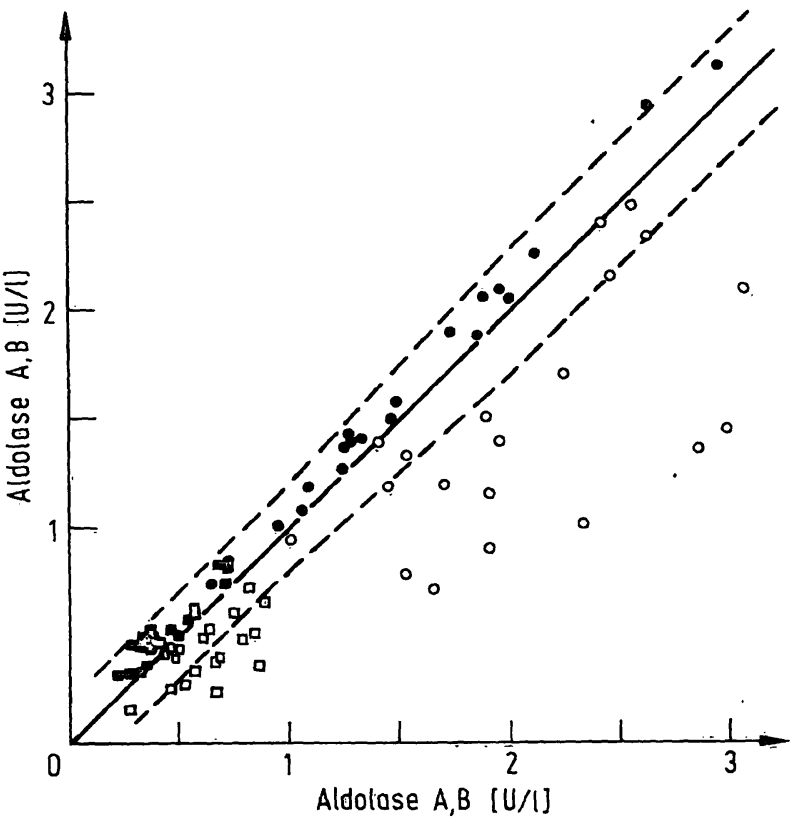

Abb. 2. Aktivitäten der Isoenzyme Aldolase $A$ und Aldolase $B$ (U/l) bei Z̈willingspaaren

Erläuterung der Darstellung șiehe Legende zu Abbildung 1

- Aldolase-A, eineiige Zwillinge

$\circ$ Aldolase-A, zweieiige Zwillinge

- Aldolase-B, eineiige Zwillinge

$\square$ Aldolase-B, zweieiige Zwillinge.

Tab. 1. Statistische Daten über die Unterschiede der Aldolase-Aktivitäten zwischen Zwilling a und Zwilling $b$ bei Paaren eineiiger bzw. zweieiiger Zwillinge ( $\mathrm{N}$ jeweils $=20$ ).

$\mathrm{EZ}=$ eineiige $\mathrm{Zwillinge}, \mathrm{ZZ}=\mathrm{zweieiige} Z$ willinge

$\triangle$ Akt. = mittlere Differenz der Aktivitäten zwischen Zwilling a und Zwilling $\mathrm{b}$ der einzelnen Paăre.

Zur Errechnung der Korrelation wurde jeweils der Meßwert des Zwillings mit höherer Aktivität = x und dẹr Mẹwert des Zwillings mit niederer Aktivität $=y$ gesetzt.

$\mathrm{s}_{\mathrm{yz}}=$ mittlere Streuung der Einzelwerte um die Korrelationsgerade.

\begin{tabular}{|c|c|c|c|c|c|c|c|c|}
\hline & \multirow{2}{*}{\multicolumn{2}{|c|}{$\Delta$ Akt. (U/l) }} & \multirow{3}{*}{$\frac{\Delta \text { Akt. ZZ }}{\Delta \text { Akt. EZ }}$} & \multirow{3}{*}{$\begin{array}{l}\text { Signifikanz der } \\
\text { Unterschiede } \\
\triangle \text { Akt. EZ zu } \\
\triangle \text { Akt. ZZ }\end{array}$} & \multicolumn{4}{|c|}{ Korrelation der Meßwerte Zwilling a/Zwilling b } \\
\hline & & & & & Korrel & Koeffizient $\mathbf{r}$ & $\mathrm{s}_{\mathrm{yx}}(1$ & \\
\hline & EZ & ZZ & & & $\mathbf{E Z}$ & $\mathbf{Z Z}$ & EZ & $\mathbf{Z Z}$ \\
\hline $\begin{array}{l}\text { Gesamt-Aldolase } \\
\text { ALD-A } \\
\text { ALD-B }\end{array}$ & $\begin{array}{l}0,14 \\
0,10 \\
0,06\end{array}$ & $\begin{array}{l}0,71 \\
0,59 \\
0,19\end{array}$ & $\begin{array}{l}5,07 \\
5,90 \\
3,16\end{array}$ & $\begin{array}{l}\mathrm{p}<0,001 \\
\mathrm{p}<0,001 \\
\mathrm{p}<0,001\end{array}$ & $\begin{array}{l}0,993 \\
0,995 \\
0,946\end{array}$ & $\begin{array}{l}0,648 \\
0,466 \\
0,589\end{array}$ & $\begin{array}{l}0,08 \\
0,05 \\
0,04\end{array}$ & $\begin{array}{l}0,54 \\
0,47 \\
0,11\end{array}$ \\
\hline
\end{tabular}


grenze identisch sind. Bei den Paaren von $\mathrm{ZZ}$ sind auch hier bei den Aktivitäten der Isoenzyme signifikante Unterschiede vorhanden. Der Unterschied zwischen Zwilling $a$ und $Z$ willing $b$ ist bei der Aldolase $A$ im Mittel bei den ZZ 6mal größer als bei den EZ, bei Aldolase B $3 \mathrm{mal}$ größer (s. auch Tab. 1).

Bezüglich der Blutgruppen und der Polymorphismen von Alanin-Transaminase, Phosphoglucomutase ( $\left.\mathrm{PGM}_{3}\right)$ $\alpha_{1}$-Antitrypsin, $C_{3}$ und Transferrin zeigte sich bei den
EZ Konkordanz, während die Phänotypen bei den ZZ teilweise differieren (Tab. 2 und 3).

\section{Danksagung}

Herrn Dr. Ehrengut danken wir für die Überlassung der Adressen der Zwillinge.

Für zuverlässige Mitarbeit danken wir Frau Nora Eckert und

Fräulein Ute Plagemann.
Tab. 2. Phänotypenbestimmung einiger Protein-Polymorphismen bei eineiigen $Z$ willingspaaren ( $Z$ willing a und $Z$ willing $b$ ). GPT = Alanin-Transaminase; PGM $_{3}=$ Phosphoglucomutase locus 3; $\alpha_{1}$ at $=\alpha_{1}$-Antitrypsin; C3 $=3$. Komponente des Komplements; $\mathrm{Tf}=$ Transfeirin .

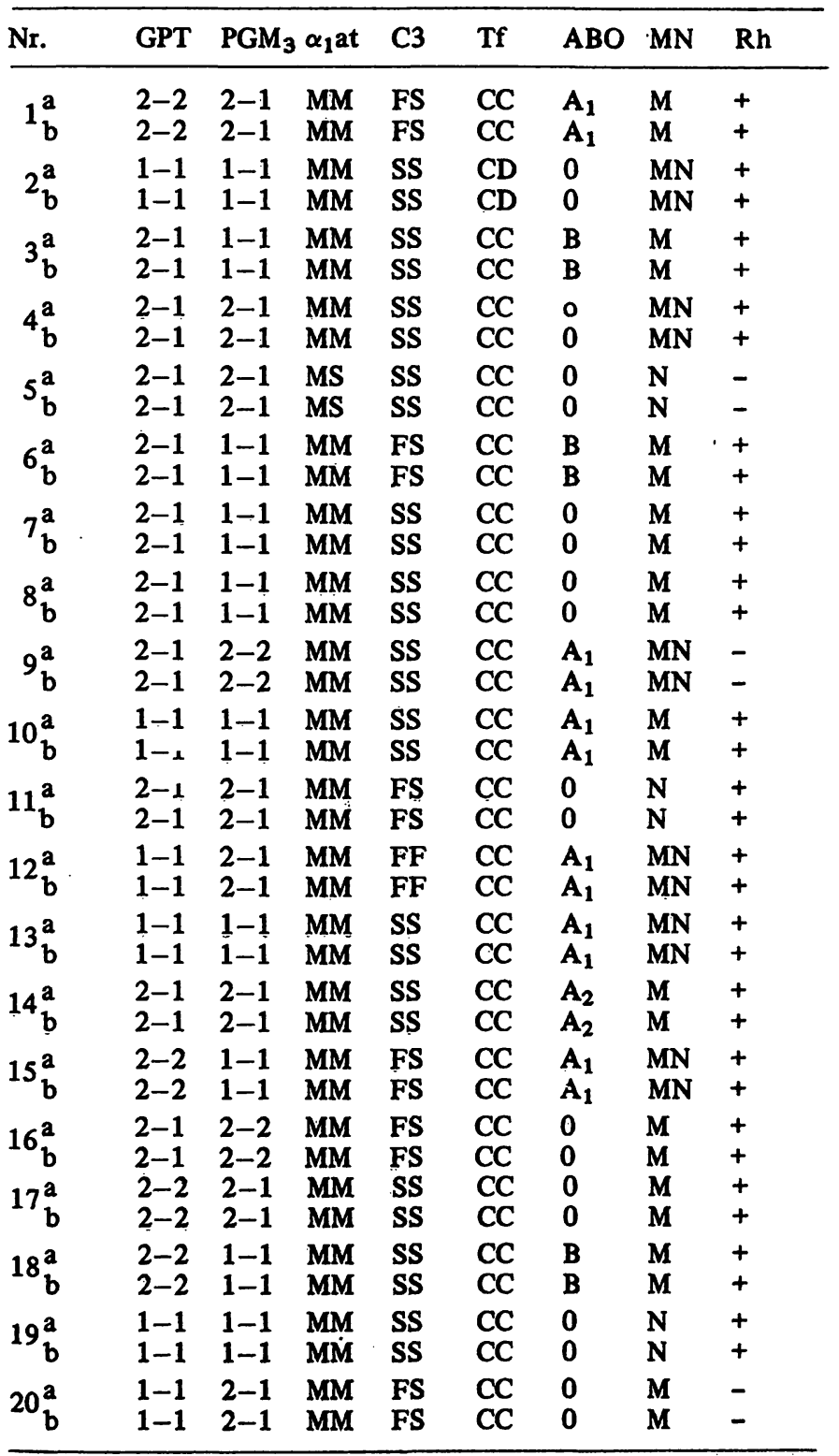

Tab. 3. Phänotypenbestimmung einiger Protein-Polymorphismen bei zweieiigen Zwillingspaaren (Zwilling a und Zwilling b). Erläuterung der Abkürzungen siehe Legende zu Tabelle 2.

\begin{tabular}{|c|c|c|c|c|c|c|c|c|}
\hline Nr. & GPT & $\mathrm{PGM}_{3}$ & $\alpha_{1}$ at & C3 & $\mathrm{Tf}$ & ABO & $\mathrm{MN}$ & $\mathbf{R h}$ \\
\hline $1_{b}^{a}$ & $\begin{array}{l}2-1 \\
2-1\end{array}$ & $\begin{array}{l}2-1 \\
1-1\end{array}$ & $\begin{array}{l}\mathrm{MM} \\
\mathrm{MM}\end{array}$ & $\begin{array}{l}\text { SS } \\
\text { SS }\end{array}$ & $\begin{array}{l}\mathrm{CC} \\
\mathrm{CC}\end{array}$ & $\begin{array}{l}A_{1} \\
B\end{array}$ & $\begin{array}{l}\mathrm{M} \\
\mathrm{MN}\end{array}$ & + \\
\hline $2_{b}^{a}$ & $\begin{array}{l}1-1 \\
1-1\end{array}$ & $\begin{array}{l}1-1 \\
2-1\end{array}$ & $\begin{array}{l}\mathrm{MM} \\
\mathrm{MM}\end{array}$ & $\begin{array}{l}\text { FS } \\
\text { SS }\end{array}$ & $\begin{array}{l}\mathrm{CC} \\
\mathrm{CC}\end{array}$ & $\begin{array}{l}0 \\
0\end{array}$ & $\begin{array}{l}\mathrm{MN} \\
\mathrm{M}\end{array}$ & + \\
\hline $3_{b}^{a}$ & $\begin{array}{l}1-1 \\
1-1\end{array}$ & $\begin{array}{l}2-1 \\
2-1\end{array}$ & $\begin{array}{l}\mathrm{MM} \\
\mathrm{MM}\end{array}$ & $\begin{array}{l}\text { FS } \\
\text { SS }\end{array}$ & $\begin{array}{l}\mathrm{CC} \\
\mathrm{CC}\end{array}$ & $\begin{array}{l}\text { B } \\
0\end{array}$ & $\begin{array}{l}\mathbf{M} \\
\mathbf{M}\end{array}$ & + \\
\hline $4_{b}^{a}$ & $\begin{array}{l}2-2 \\
2-2\end{array}$ & $\begin{array}{l}2-1 \\
2-1\end{array}$ & $\begin{array}{l}\text { MM } \\
\text { MM }\end{array}$ & $\begin{array}{l}\text { SS } \\
\text { SS }\end{array}$ & $\begin{array}{l}\mathrm{CC} \\
\mathrm{CC}\end{array}$ & $\begin{array}{l}0 \\
0\end{array}$ & $\begin{array}{l}\mathbf{M} \\
\mathbf{M}\end{array}$ & - \\
\hline $5_{\mathrm{b}}^{\mathrm{a}}$ & $\begin{array}{l}2-2 \\
2-1\end{array}$ & $\begin{array}{l}1-1 \\
1-1\end{array}$ & $\begin{array}{l}\text { MM } \\
\text { MM }\end{array}$ & $\begin{array}{l}\text { SS } \\
\text { SS }\end{array}$ & $\begin{array}{l}\mathrm{CC} \\
\mathrm{CC}\end{array}$ & $\begin{array}{l}A_{1} \\
0\end{array}$ & $\begin{array}{l}\mathrm{MN} \\
\mathrm{M}\end{array}$ & + \\
\hline $6_{\mathrm{b}}^{\mathrm{a}}$ & $\begin{array}{l}2-1 \\
2-1\end{array}$ & $\begin{array}{l}1-1 \\
1-1\end{array}$ & $\begin{array}{l}\mathbf{M M} \\
\mathbf{M M}\end{array}$ & $\begin{array}{l}\text { SS } \\
\text { FS }\end{array}$ & $\begin{array}{l}\mathrm{CC} \\
\mathrm{CC}\end{array}$ & $\begin{array}{l}\mathbf{A}_{2} \\
\mathbf{A}_{1}\end{array}$ & $\begin{array}{l}\mathrm{MN} \\
\mathrm{N}\end{array}$ & + \\
\hline $7_{\mathrm{b}}^{\mathrm{a}}$ & $\begin{array}{l}2-2 \\
2-1\end{array}$ & $\begin{array}{l}1-1 \\
2-1\end{array}$ & $\begin{array}{l}\text { MM } \\
\text { MM }\end{array}$ & $\begin{array}{l}\text { SS } \\
\text { SS }\end{array}$ & $\begin{array}{l}\mathrm{CC} \\
\mathrm{CC}\end{array}$ & $\begin{array}{l}0 \\
0\end{array}$ & $\begin{array}{l}\mathbf{N} \\
\mathbf{N}\end{array}$ & + \\
\hline $8_{b}^{a}$ & $\begin{array}{l}2-1 \\
2-1\end{array}$ & $\begin{array}{l}1-1 \\
2-1\end{array}$ & $\begin{array}{l}\text { MM } \\
\mathbf{M M}\end{array}$ & $\begin{array}{l}\text { SS } \\
\text { SS }\end{array}$ & $\begin{array}{l}\mathrm{CC} \\
\mathrm{CC}\end{array}$ & $\begin{array}{l}0 \\
0\end{array}$ & $\begin{array}{l}M \\
M\end{array}$ & + \\
\hline $9_{b}^{a}$ & $\begin{array}{l}2-1 \\
2-2\end{array}$ & $\begin{array}{l}1-1 \\
1-1\end{array}$ & $\begin{array}{l}\text { MM } \\
\text { MM }\end{array}$ & $\begin{array}{l}\text { SS } \\
\text { FS }\end{array}$ & $\begin{array}{l}\mathrm{CC} \\
\mathrm{CC}\end{array}$ & $\begin{array}{l}0 \\
\text { B }\end{array}$ & $\begin{array}{l}\mathbf{M} \\
\mathbf{M}\end{array}$ & $\begin{array}{l}+ \\
-\end{array}$ \\
\hline $10_{\mathrm{b}}^{\mathrm{a}}$ & $\begin{array}{l}2-1 \\
2-1\end{array}$ & $\begin{array}{l}1-1 \\
1-1\end{array}$ & $\begin{array}{l}\text { MM } \\
\text { MM }\end{array}$ & $\begin{array}{l}\text { SS } \\
\text { SS }\end{array}$ & $\begin{array}{l}\mathrm{CC} \\
\mathrm{CC}\end{array}$ & $\begin{array}{l}0 \\
A_{1}\end{array}$ & $\begin{array}{l}\mathrm{MN} \\
\mathbf{N}\end{array}$ & $\begin{array}{l}+ \\
+\end{array}$ \\
\hline $11_{b}^{a}$ & $\begin{array}{l}2-1 \\
1-1\end{array}$ & $\begin{array}{l}2-1 \\
1-1\end{array}$ & $\begin{array}{l}\mathrm{MM} \\
\mathrm{MM}\end{array}$ & $\begin{array}{l}\text { FS } \\
\text { FS }\end{array}$ & $\begin{array}{l}\mathrm{CC} \\
\mathrm{CC}\end{array}$ & $\begin{array}{l}A_{1} \\
0\end{array}$ & $\begin{array}{l}\text { MN } \\
\text { MN }\end{array}$ & $\begin{array}{l}+ \\
-\end{array}$ \\
\hline & $\begin{array}{l}1-1 \\
1-1\end{array}$ & $\begin{array}{l}2-2 \\
2-2\end{array}$ & $\begin{array}{l}\mathrm{MM} \\
\mathrm{MZ}\end{array}$ & $\begin{array}{l}\text { SS } \\
\text { FS }\end{array}$ & $\begin{array}{l}\mathrm{CC} \\
\mathrm{CC}\end{array}$ & $\begin{array}{l}0 \\
0\end{array}$ & $\begin{array}{l}\mathrm{M} \\
\mathrm{MN}\end{array}$ & $\overline{+}$ \\
\hline & $\begin{array}{l}1-1 \\
2-1\end{array}$ & $\begin{array}{l}1-1 \\
.2-1\end{array}$ & $\begin{array}{l}\text { MM } \\
\text { MM }\end{array}$ & $\begin{array}{l}\text { SS } \\
\text { SS }\end{array}$ & $\begin{array}{l}\mathrm{CC} \\
\mathrm{CC}\end{array}$ & $\begin{array}{l}0 \\
\mathbf{A}_{1}\end{array}$ & $\begin{array}{l}\text { MN } \\
\text { MN }\end{array}$ & + \\
\hline & $\begin{array}{l}1-1 \\
1-1\end{array}$ & $\begin{array}{l}2-1 \\
2-1\end{array}$ & $\begin{array}{l}\text { MM } \\
\text { MM }\end{array}$ & $\begin{array}{l}\text { FF } \\
\text { FS }\end{array}$ & $\begin{array}{l}\mathrm{CC} \\
\mathrm{CC}\end{array}$ & $\begin{array}{l}A_{1} \\
A_{1}\end{array}$ & $\begin{array}{l}\mathbf{M} \\
\mathbf{M}\end{array}$ & $\begin{array}{l}+ \\
+\end{array}$ \\
\hline & $\begin{array}{l}2-1 \\
2-1\end{array}$ & $\begin{array}{l}2-1 \\
2-1\end{array}$ & $\begin{array}{l}\text { MM } \\
\text { MM }\end{array}$ & $\begin{array}{l}\text { SS } \\
\text { SS }\end{array}$ & $\begin{array}{l}\mathrm{CC} \\
\mathrm{B}_{2} \mathrm{C}\end{array}$ & $\begin{array}{l}\text { B } \\
\text { B }\end{array}$ & $\begin{array}{l}\mathrm{M} \\
\mathrm{MN}\end{array}$ & + \\
\hline $16_{\mathrm{b}}^{\mathrm{a}}$ & $\begin{array}{l}2-1 \\
2-1\end{array}$ & $\begin{array}{l}1-1 \\
1-1\end{array}$ & $\begin{array}{l}\text { MM } \\
\text { MM }\end{array}$ & $\begin{array}{l}\text { FS } \\
\text { FS }\end{array}$ & $\begin{array}{l}\mathrm{CC} \\
\mathrm{CC}\end{array}$ & $\begin{array}{l}0 \\
0\end{array}$ & $\begin{array}{l}\mathbf{N} \\
\mathbf{N}\end{array}$ & $\overline{+}$ \\
\hline $17_{\mathrm{b}}^{\mathrm{a}}$ & $\begin{array}{l}2-1 \\
1-1\end{array}$ & $\begin{array}{l}1-1 \\
2-1\end{array}$ & $\begin{array}{l}\mathbf{M M} \\
\mathbf{M M}\end{array}$ & $\begin{array}{l}\text { SS } \\
\text { FF }\end{array}$ & $\begin{array}{l}\mathrm{CC} \\
\mathrm{CC}\end{array}$ & $\begin{array}{l}0 \\
0\end{array}$ & $\begin{array}{l}\mathrm{MN} \\
\mathrm{N}\end{array}$ & + \\
\hline $18_{b}^{a}$ & $\begin{array}{l}1-1 \\
2-2\end{array}$ & $\begin{array}{l}1-1 \\
1-1\end{array}$ & $\begin{array}{l}\text { MM } \\
\mathbf{M M}\end{array}$ & $\begin{array}{l}\text { SS } \\
\text { FS }\end{array}$ & $\begin{array}{l}\mathrm{CC} \\
\mathrm{CC}\end{array}$ & $\begin{array}{l}0 \\
0\end{array}$ & $\mathbf{M}$ & + \\
\hline $19^{a}$ & $\begin{array}{l}2-1 \\
2-1\end{array}$ & $\begin{array}{l}2-1 \\
2-1\end{array}$ & $\begin{array}{l}\mathrm{MM} \\
\mathbf{M M}\end{array}$ & $\begin{array}{l}\text { SS } \\
\text { SS }\end{array}$ & $\begin{array}{l}\mathrm{CC} \\
\mathrm{CC}\end{array}$ & $\begin{array}{l}0 \\
0\end{array}$ & $\begin{array}{l}\mathrm{M} \\
\mathrm{M}\end{array}$ & + \\
\hline $20 \frac{a}{b}$ & $\begin{array}{l}2-1 \\
1-1\end{array}$ & $\begin{array}{l}2-1 \\
2-1\end{array}$ & $\begin{array}{l}\mathrm{MM} \\
\mathrm{MM}\end{array}$ & $\begin{array}{l}\text { SS } \\
\text { FS }\end{array}$ & $\begin{array}{l}\mathrm{CC} \\
\mathrm{CC}\end{array}$ & $\begin{array}{l}0 \\
0\end{array}$ & $\begin{array}{l}\text { M } \\
\text { MN }\end{array}$ & $\begin{array}{l}+ \\
+\end{array}$ \\
\hline
\end{tabular}




\section{I.jerafur}

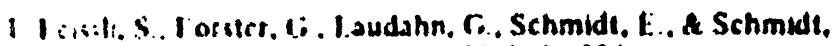
I W. 11406) Klin Wochinuhr. 4. 390.396.

: Cioridd: II. W . A Bcnkmann. H. (i. (1972) Humangenelik $15.27727 \mathrm{~K}$.

3. (misilit. II. W.. Stahn. M. (1974) Ilumangenetik 22. $2+1$ 245.
4. Foperhol, M. K. \& Laurell, C.-B. (1967) Clin. Chim. Acta J6. 199-203.

5. Teisberg. P. (1970) Vox. Sang 19.47.

6. Wurzburg. U., Wile. I., Hennrich, N. \& Lang. H. (1974) diese 2. 12,176-177.
Professar Dr. H. W. Coedde Instifut fir Humangenetik der Untversitàt D-2000 Hamburg S4, Butcnfeld 32

Federal Republic of Germany 